\title{
Bivariate Segment Approximation and Splines
}

G. Meinardus, G. Nürnberger, G. Walz

Reihe Mathematik Nr. 206/1996) 
Key words: Bivariate approximation, Segment approximation, Bivariate splines, Functionals.

AMS(MOS) subject classification: 41A15, 41A63, 65D07.

Running title: Bivariate Segment Approximation. 
Abstract. The problem to determine partitions of a given rectangle which are optimal for segment approximation (e.g. by bivariate piecewise polynomials) is investigated. We give criteria for optimal partitions and develop algorithms for computing optimal partitions of certain types. It is shown that there is a surprising relationship between various types of optimal partitions. In this way, we obtain good partitions for interpolation by tensor product spline spaces. Our numerical examples show that the methods work efficiently. 


\section{Introduction}

The motivation for our investigations comes from the following problem. Let a rectangle $\Omega=[a, b] \times[c, d]$ be subdivided by a fixed number of horizontal and vertical lines. Such a partition is called of type 3 (see Figure 3). We interpolate a function $f \in C(\Omega)$ by a function $A(f)$ from some tensor product spline space defined on this partition. The problem is to determine a partition of $\Omega$ for which the error $\|f-A(f)\|_{\Omega}$ is relatively small.

Since no general criteria are available for good or optimal partitions of this type, we try to get such partitions via piecewise polynomial approximation. Therefore, we consider the following general subdivision problem. Let a partition of $\Omega$ into a fixed number of subrectangles $\left\{\Omega_{\mu, \nu}\right\}$ as in Figure 1 or Figure 2 be given. Such a partition $\left\{\Omega_{\mu, \nu}\right\}$ is called of type 1 or type 2 , respectively. Moreover, let a functional $d$ be given which associates to each subrectangle $\Omega_{\mu, \nu}$ a real number $d\left(\Omega_{\mu, \nu}\right)$. We first investigate the problem to determine a partition of type 1 respectively type 2 for which $\max _{\mu, \nu} d\left(\Omega_{\mu, \nu}\right)$ is as small as possible. Such a partition is called optimal.

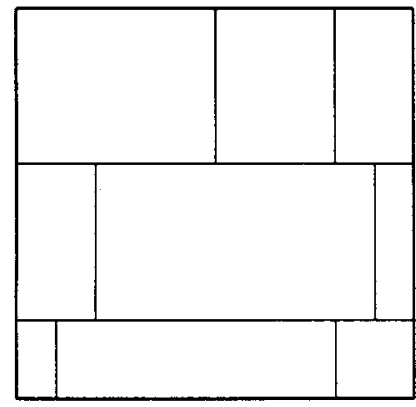

Figure 1. Partition of type 1

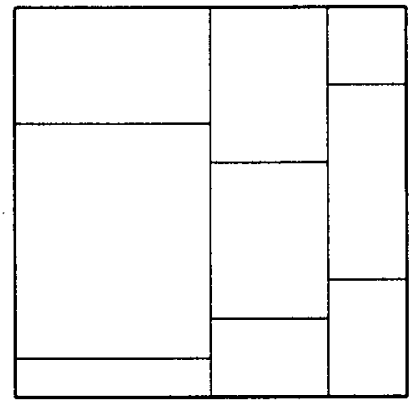

Figure 2. Partition of type 2

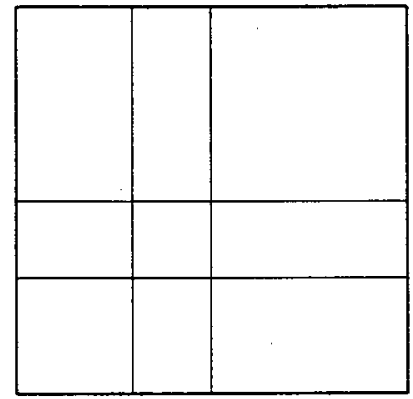

Figure 3. Partition of type 3

For a general class of functionals $d$ (e.g. the minimal deviation for piecewise polynomials), we develop criteria for optimal partitions of type 1 respectively type 2 (Theorem 2.2). Furthermore, we describe an algorithm for computing a partition for which all values $d\left(\Omega_{\mu, \nu}\right)$ 
are the same. Such a partition is shown to be optimal. In the univariate case, similar methods were developed by Nürnberger, Sommer \& Strauß [13] and Meinardus, Nürnberger, Sommer \& Strauß [9]. However, the bivariate case is more complex, since certain anomalies may occur. (For further results on univariate segment approximation see e.g. [1], [4], [5], $[6],[7],[8],[11],[12],[15]$, and the references therein.)

Partitions of type 1 and 2 are more general than partitions of type 3 . Therefore, it is surprising that for certain functionals $d$, the optimal value of type 3 partitions is equal to one of the optimal values of type 1 and type 2 partitions (Theorem 3.5). Moreover, it is shown that in this case, the horizontal lines of an optimal type 1 partition combined with the vertical lines of an optimal type 2 partition yield an optimal partition of type 3 (Theorem 3.6).

A functional $d$ for which these results hold, is given by

$$
d(R)=\max \left\{\left\|f_{x^{m+1}}\right\|\left(\xi_{2}-\xi_{1}\right)^{m+1},\left\|f_{y^{m+1}}\right\|\left(\eta_{2}-\eta_{1}\right)^{m+1}\right\},
$$

where $R=\left[\xi_{1}, \xi_{2}\right] \times\left[\eta_{1}, \eta_{2}\right]$. This functional reflects the error for interpolation by the tensor product polynomial space $\Pi_{m} \otimes \Pi_{m}$ (see Theorem 3.2 and (3.3)). Moreover, the numerical tests show that our method also works efficiently if we use the functional

$$
\tilde{d}(R)=\|f-P(f)\|_{R}
$$

where $P(f) \in \Pi_{m} \otimes \Pi_{m}$ interpolates $f$ at $(m+1)^{2}$ uniformly distributed points in $R$ (although $\widetilde{d}$ does not quite fit into the general setting of our results). Finally, some numerical examples indicate that the optimal partitions of type 3 for piecewise polynomial approximation yield good partitions for interpolation by tensor product spline spaces.

\section{Partitions of Type 1 and Type 2}

In this section, we give results on the existence of optimal partitions of type 1 and type 2 and derive criteria for optimality. Building on these results, we describe an algorithm for computing optimal partitions.

Let a rectangle $\Omega=[a, b] \times[c, d]$ be given. We subdivide $\Omega$ into $r$ horizontal strips

$$
J_{\mu}:=[a, b] \times\left[\tau_{\mu-1}, \tau_{\mu}\right]
$$

where $c=\tau_{0} \leq \tau_{1} \leq \cdots \leq \tau_{r-1} \leq \tau_{r}=d$. Furthermore, let each strip $J_{r}$ be subdivided into $k$ subrectangles

$$
\Omega_{\mu, \nu}=\left[\sigma_{\mu, \nu-1}, \sigma_{\mu, \nu}\right] \times\left[\tau_{\mu-1}, \tau_{\mu}\right]
$$

where $a=\sigma_{\mu, 0} \leq \sigma_{\mu, 1} \leq \cdots \leq \sigma_{\mu, k-1} \leq \sigma_{\mu, k}=b$. A partition $\left\{\Omega_{\mu, \nu}\right\}$ of this type is called a partition of type 1 (see Figure 1). 
If we subdivide $\Omega$ into $k$ vertical strips and each strip into $r$ subrectangles, then the resulting partition is called of type 2 (see Figure 2). If $\Omega$ is subdivided into $r \cdot k$ rectangles of the form

$$
\Omega_{\mu, \nu}=\left[\sigma_{\nu-1}, \sigma_{\nu}\right] \times\left[\tau_{\mu-1}, \tau_{\mu}\right]
$$

where $a=\sigma_{0} \leq \sigma_{1} \leq \cdots \leq \sigma_{k-1} \leq \sigma_{k}=b$, then the partition $\left\{\Omega_{\mu, \nu}\right\}$ is called of type 3 (see Figure 3).

Let $M$ denote the set of all subrectangles $R=\left[\xi_{1}, \xi_{2}\right] \times\left[\eta_{1}, \eta_{2}\right]$ of $\Omega$. We consider a functional $d$, which associates to each $R \in M$ a real number $d(R)$ with the following properties:

$$
\begin{array}{ll}
d(R) \leq d(\widetilde{R}), & \text { if } R \subset \widetilde{R}, R, \widetilde{R} \in M, \\
d(R)=0, & \text { if } R \text { consists of one single point }, \\
d\left(R_{n}\right) \rightarrow d(R), & \text { if } R_{n} \rightarrow R, R, R_{n} \in M \text { for all } n .
\end{array}
$$

In contrast to the univariate case (see [9]), $d(R)$ may be strictly positive, although the rectangle $R$ degenerates to a line segment.

Definition 2.1. Fix a number $i \in\{1,2,3\}$. A partition $\left\{\Omega_{\mu, \nu}\right\}$ of type $i$ is called optimal if

$$
\max _{\mu, \nu} d\left(\Omega_{\mu, \nu}\right)=\inf _{\left\{\tilde{\Omega}_{\mu, \nu}\right\}} \max _{\mu, \nu} d\left(\widetilde{\Omega}_{\mu, \nu}\right)
$$

where the infimum is taken over all partitions $\left\{\widetilde{\Omega}_{\mu, \nu}\right\}$ of type $i$. For an optimal partition of type $i$, the value $m_{i}=\max _{\mu, \nu} d\left(\Omega_{\mu}, \nu\right)$ is called the optimal value. A partition $\left\{\Omega_{\mu, \nu}\right\}$ of type $i$ is called leveled, if all values $d\left(\Omega_{\mu, \nu}\right), \mu=1, \ldots, r, \nu=1, \ldots, k$, are the same.

We first prove a result on optimal partitions of type 1 and 2 .

Theorem 2.2. For fixed $i \in\{1,2\}$, the following statements hold:

(i) For every partition $\left\{\Omega_{\mu, \nu}\right\}$ of type $i$,

$$
\min _{\mu, \nu} d\left(\Omega_{\mu, \nu}\right) \leq m_{i} \leq \max _{\mu, \nu} d\left(\Omega_{\mu, \nu}\right)
$$

(ii) Every leveled partition of type $i$ is optimal.

(iii) $A$ leveled partition of type $i$ exists.

(iv) If $d(R)<d(\widetilde{R})$ for all $R \subsetneq \widetilde{R}, R, \widetilde{R} \in M$, then a unique optimal partition of type $i$ exists.

Proof. It suffices to consider the case when $i=1$. Since the mapping which associates to each set of real numbers $\left\{\sigma_{\mu, \nu}, \tau_{\mu}\right\}$ (cf. (2.2)) the real number $\max _{\mu, \nu} d\left(\Omega_{\mu, \nu}\right)$ is continuous, it 
follows from compactness arguments that the infimum in (2.7) is attained. Therefore, an optimal partition exists.

To prove statement (i), we first have to show the following. Let arbitrary type 1 partitions $\left\{\Omega_{\mu, \nu}\right\}$ and $\left\{\widetilde{\Omega}_{\mu, \nu}\right\}$ with $\left\{\Omega_{\mu, \nu}\right\} \neq\left\{\widetilde{\Omega}_{\mu, \nu}\right\}$ be given. Then

$$
\text { there is an } \Omega_{\mu, \nu} \text { with } \Omega_{\mu, \nu} \subsetneq \widetilde{\Omega}_{\mu, \nu} .
$$

To show this, we distinguish two cases.

Case 1. $\widetilde{\tau}_{\mu}=\tau_{\mu}$ for $\mu=0, \ldots, r$

Since $\left\{\Omega_{\mu, \nu}\right\} \neq\left\{\widetilde{\Omega}_{\mu, \nu}\right\}$, there is an index $m$ such that $\left\{\sigma_{m, \nu}\right\} \neq\left\{\widetilde{\sigma}_{m, \nu}\right\}$. We consider the strip $[a, b] \times\left[\tau_{m-1}, \tau_{m}\right]$. By running through $\sigma_{m, 0}, \ldots, \sigma_{m, k}$ it follows that there is an index $n$ with $\left[\sigma_{m, n-1}, \sigma_{m, n}\right] \subsetneq\left[\widetilde{\sigma}_{m, n-1}, \widetilde{\sigma}_{m, n}\right]$, hence $\Omega_{m, n} \subsetneq \widetilde{\Omega}_{m, n}$.

Case 2. $\widetilde{\tau}_{\mu} \neq \tau_{\mu}$ for some $\mu \in\{1, \ldots, r-1\}$

By running through $\tau_{0}, \tau_{1}, \ldots, \tau_{r}$, it follows that there is an index $m$, such that

$$
\left[\tau_{m-1}, \tau_{m}\right] \subsetneq\left[\tilde{\tau}_{m-1}, \tilde{\tau}_{m}\right]
$$

We consider the strips $[a, b] \times\left[\widetilde{\tau}_{m-1}, \widetilde{\tau}_{m}\right]$ and $[a, b] \times\left[\tau_{m-1}, \tau_{m}\right]$. It follows that there is an index $n$ with $\left[\sigma_{m, n-1}, \sigma_{m, n}\right] \subset\left[\widetilde{\sigma}_{m, n-1}, \widetilde{\sigma}_{m, n}\right]$, hence $\Omega_{m, n} \subsetneq \widetilde{\Omega}_{m, n}$. This proves statement (2.8).

It is obvious that $m_{1} \leq \max _{\mu, \nu} d\left(\Omega_{\mu, \nu}\right)$. Now, assume that $\min _{\mu, \nu} d\left(\Omega_{\mu, \nu}\right)>m_{1}$. Let $\left\{\widetilde{\Omega}_{\mu, \nu}\right\}$ be an optimal partition. Then by (2.8)

$$
m_{1} \geq d\left(\widetilde{\Omega}_{\mu, \nu}\right) \geq d\left(\Omega_{\mu, \nu}\right)>m_{1}
$$

for some $(\mu, \nu)$, which is a contradiction. This shows (i). Statement (ii) is an immediate consequence of (i). We now prove (iii). Let us consider an arbitrary strip

$$
J_{\mu}=[a, b] \times\left[\tau_{\mu-1}, \tau_{\mu}\right]
$$

For $a \leq \xi_{1} \leq \xi_{2} \leq b$, we set

$$
\Omega\left(\xi_{1}, \xi_{2}\right):=\left[\xi_{1}, \xi_{2}\right] \times\left[\tau_{\mu-1}, \tau_{\mu}\right]
$$

Claim 1. Real numbers

$$
a=\sigma_{\mu, 0} \leq \sigma_{\mu, 1} \leq \cdots \leq \sigma_{\mu, k}=b
$$

exist such that

$$
d\left(\Omega\left(\sigma_{\mu, \nu}, \sigma_{\mu, \nu+1}\right)\right)=d\left(\Omega\left(\sigma_{\mu, \nu+1}, \sigma_{\mu, \nu+2}\right)\right) \quad \text { for } \nu=0, \ldots, k-2,
$$

i.e., a leveled partition of the $\operatorname{strip} J_{\mu}$.

We prove this claim by induction on $k$, so first assume $k=1$. For any $\xi \in[a, b]$, we set

$$
c(\xi):=d(\Omega(a, \xi))-d(\Omega(\xi, b)) .
$$


It follows from (2.4) that

$$
c(a) \leq 0 \quad \text { and } \quad c(b) \geq 0 .
$$

Therefore, the continuity of $c$ (cf. (2.6)) implies the existence of a number $\xi=: \sigma_{\mu, 1} \in[a, b]$ with $c\left(\sigma_{\mu, 1}\right)=0$. This proves Claim 1 in the case $k=1$. (Note that, in contrast to the univariate case, it may happen $\sigma_{\mu, 1}=a$ or $\sigma_{\mu, 1}=b$, due to the fact that $d(\Omega(a, a))$ and $d(\Omega(b, b))$ are strictly positive in general.)

Now we assume that Claim 1 is true for $k-1$. We define, for $\xi \in[a, b]$, functionals $c_{1}$ and $c_{2}$ by

$$
c_{1}(\xi):=\min \left\{\max _{0 \leq \nu \leq k-1} d\left(\Omega\left(\tilde{\sigma}_{\mu, \nu}, \tilde{\sigma}_{\mu, \nu+1}\right)\right): a=\tilde{\sigma}_{\mu, 0} \leq \widetilde{\sigma}_{\mu, 1} \leq \cdots \leq \tilde{\sigma}_{\mu, k-1}:=\xi\right\}
$$

and

$$
c_{2}(\xi):=c_{1}(\xi)-d(\Omega(\xi, b))
$$

Again by the continuity of the functional $c_{2}$, there is some $\xi:=\sigma_{\mu, k-1}$ with

$$
c_{2}\left(\sigma_{\mu, k-1}\right)=0
$$

Moreover, by the induction hypothesis there exists a leveled partition with parameters $\sigma_{\mu, 0}, \ldots, \sigma_{\mu, k-1}$ for $k-1$. It follows from (i) that this partition is also optimal, and so

$$
d\left(\Omega\left(\sigma_{\mu, \nu}, \sigma_{\mu, \nu+1}\right)\right)=c_{1}\left(\sigma_{\mu, k-1}\right) \quad \text { for } \nu=0, \ldots, k-2 .
$$

Finally, a combination of statements (2.11), (2.12) and (2.13) completes the proof of Claim 1.

Now, we set for $c \leq \eta_{1} \leq \eta_{2} \leq d$,

$$
I\left(\eta_{1}, \eta_{2}\right):=[a, b] \times\left[\eta_{1}, \eta_{2}\right] .
$$

By Claim 1 we know that there is a leveled partition $\left\{R_{\nu}\left(\eta_{1}, \eta_{2}\right)\right\}_{\nu=1, \ldots, k}$ of this strip, which minimizes the functional

$$
\max _{\nu} d\left(\widetilde{R}_{\nu}\left(\eta_{1}, \eta_{2}\right)\right)
$$

over all partitions $\left\{\widetilde{R}_{\nu}\left(\eta_{1}, \eta_{2}\right)\right\}_{\nu=1, \ldots, k}$ of the strip $I\left(\eta_{1}, \eta_{2}\right)$. Let $\bar{d}\left(I\left(\eta_{1}, \eta_{2}\right)\right):=d\left(R_{1}\left(\eta_{1}, \eta_{2}\right)\right)$.

Claim 2. Real numbers

$$
c=\tau_{0} \leq \tau_{1} \leq \cdots \leq \tau_{\nu-1} \leq \tau_{\nu}=d
$$

exist such that

$$
\bar{d}\left(I\left(\tau_{\mu}, \tau_{\mu+1}\right)\right)=\bar{d}\left(I\left(\tau_{\mu+1}, \tau_{\mu+2}\right)\right) \quad \text { for } \mu=0, \ldots, r-2 .
$$


Claim 2 follows analogously as Claim 1 . This proves (iii).

Now, we prove (iv). From (iii) we know that a leveled partition $\left\{\Omega_{\mu, \nu}\right\}$ exists. Assume that there is another optimal partition $\left\{\widetilde{\Omega}_{\mu, \nu}\right\}$. Then, by (2.8), there is an $\widetilde{\Omega}_{\mu, \nu}$ such that $\Omega_{\mu, \nu} \subsetneq \widetilde{\Omega}_{\mu, \nu}$. But since $\left\{\Omega_{\mu, \nu}\right\}$ is leveled, this implies

$$
m_{1}=d\left(\Omega_{\mu, \nu}\right)<d\left(\widetilde{\Omega}_{\mu, \nu}\right),
$$

which contradicts the optimality of $\left\{\widetilde{\Omega}_{\mu, \nu}\right\}$.

Before describing an algorithm for computing leveled partitions, we investigate special functionals $d$ which arise from best approximation.

Let $G$ be a subspace of $C(R)$, where $R=\left[\xi_{1}, \xi_{2}\right] \times\left[\eta_{1}, \eta_{2}\right]$ and $f \in C(R)$ be given. A function $g_{f} \in G$ is called best approximation of $f$ if $\left\|f-g_{f}\right\|_{R}=\inf _{g \in G}\|f-g\|_{R}$, where $\|h\|_{R}=\max _{z \in R}|h(z)|$ for $h \in C(R)$. The value $\rho(f, G)_{R}=\inf _{g \in G}\|f-g\|_{R}$ is called minimal deviation of $f$ from $G$.

In the next two examples, we investigate functionals $d$ of the form $d(R):=d(f, R):=$ $\rho(f, G)_{R}$.

Example 2.3. We denote by $\widetilde{\Pi}_{m}=\operatorname{span}\left\{x^{\mu} y^{\nu}: \mu, \nu \in \mathbb{N}_{0}, \mu, \nu \leq m\right\}$ the space of bivariate polynomials of total degree $m$. Given the function $f(x, y)=x^{m_{1}} y^{m_{2}}$, we define the functional $d$ by

$$
d(R):=d(f, R):=\rho\left(f, \widetilde{\Pi}_{m_{1}+m_{2}-1}\right)
$$

for all $R=\left[\xi_{1}, \xi_{2}\right] \times\left[\eta_{1}, \eta_{2}\right]$ in $\Omega=[a, b] \times[c, d]$. We will show that

$$
d(R)=\frac{\left(\xi_{2}-\xi_{1}\right)^{m_{1}}\left(\eta_{2}-\eta_{1}\right)^{m_{2}}}{2^{2 m_{1}+2 m_{2}-2}} .
$$

Then it is easy to verify that for $d$, the equidistant partition is the unique optimal partition of type 1 , type 2 respectively type 3 with $m_{1}=m_{2}=m_{3}=\frac{(b-a)^{m_{1}}(d-c)^{m_{2}}}{2^{2 m_{1}+2 m_{2}-2 \cdot k^{m_{1}} \cdot r^{m_{2}}}}$.

We first determine $d(R)$ for $R_{0}=[-1,1] \times[-1,1]$. Generalizing earlier work of Ehlich $\&$ Zeller [3], it was shown by Reimer [14] that a best approximation $p_{f} \in \widetilde{\Pi}_{m_{1}+m_{2}-1}$ of $f$ exists such that

$$
f(x, y)-p_{f}(x, y)=\frac{1}{2^{m_{1}+m_{2}-2}} T_{m_{1}}(x) T_{m_{2}}(y),
$$

where $T_{m}$ denotes the univariate Chebyshev polynomial of the first kind of degree $m$ (see e.g. [6]). Since $\left\|T_{m}\right\|_{[1,1]}=1$, we get $\rho\left(f, \Pi_{m_{1}+m_{2}-1}\right)_{R_{0}}=\frac{1}{2^{m_{1}+m_{2}-2}}$. By transforming $R_{0}$ to $R$, we get (2.15).

Example 2.4. For $\Omega=[0,1] \times[0,1]$ and $f(x, y)=\sqrt{x y}$, we consider the functional $d(R)=$ $d(f, R)=\rho\left(f, \widetilde{\Pi}_{1}\right)_{R}$. We will determine an explicit expression for a leveled type 1 partition.

We first prove the following 
Claim. The polynomial

$$
\begin{array}{r}
p_{f}(x, y)=\frac{1}{4\left(\sqrt{\xi_{1}}+\sqrt{\xi_{2}}\right)\left(\sqrt{\eta_{1}}+\sqrt{\eta_{2}}\right)}\left(2\left(\sqrt{\eta_{1}}+\sqrt{\eta_{2}}\right)^{2} x+2\left(\sqrt{\xi_{1}}+\sqrt{\xi_{2}}\right)^{2} y\right. \\
\left.-\left(\sqrt{\xi_{2} \eta_{2}}-\sqrt{\xi_{1} \eta_{1}}\right)^{2}\right)
\end{array}
$$

is a best approximation of $f$ from $\widetilde{\Pi}_{1}$ on $R=\left[\xi_{1}, \xi_{2}\right] \times\left[\eta_{1}, \eta_{2}\right]$ and the corresponding minimal deviation is

$$
d(R)=\frac{\left(\sqrt{\xi_{2} \eta_{2}}-\sqrt{\xi_{1} \eta_{1}}\right)^{2}}{4\left(\sqrt{\xi_{1}}+\sqrt{\xi_{2}}\right)\left(\sqrt{\eta_{1}}+\sqrt{\eta_{2}}\right)} .
$$

This can be seen as follows. Let $H=f-p_{f}$ be the error function. Then the set of extremal points of $H$ consists of the two points $\left(\xi_{1}, \eta_{2}\right)$ and $\left(\xi_{2}, \eta_{1}\right)$, where $H$ attains the value $-d(R)$, and of all points of the intersection of the line

$$
\left(\sqrt{\eta_{1}}-\sqrt{\eta_{2}}\right)^{2} x-\left(\sqrt{\xi_{1}}+\sqrt{\xi_{2}}\right)^{2} y=0
$$

with the rectangle $R$, where $H$ attains the value $d(R)$. Since there is no function in $\widetilde{\Pi}_{1}$ which is negative at the points $\left(\xi_{1}, \eta_{2}\right)$ and $\left(\xi_{2}, \eta_{1}\right)$ and positive on the intersection of the above line with $R$, it follows by Kolmogorov's criterion (see e.g. [6]) that $p_{f}$ is a best approximation of $f$, and that $d(R)$ is the corresponding minimal deviation.

In order to compute a leveled partition, we recursively define functions $g_{\nu}:[0,1] \rightarrow \mathbb{R}$ for $\nu \in \mathbb{N}_{0}$. We set

$$
g_{0}(\lambda)=0 \quad \text { for all } \lambda \in[0,1]
$$

and

$$
g_{\nu+1}(\lambda)=\frac{1}{2}+\lambda g_{\nu}(\lambda)+\sqrt{\frac{1}{4}+(1+\lambda) g_{\nu}(\lambda)}
$$

for $\nu \in \mathbb{N}_{0}$. It is easy to verify that

(i) $g_{\nu}$ is continuous and monotone on $[0,1]$;

$$
\begin{aligned}
& g_{\nu}(1)=\frac{\nu(\nu+1)}{2} ; \\
& g_{\nu}(\lambda)<g_{\nu+1}(\lambda)<\frac{2}{(1-\lambda)^{2}} \quad \text { for } \lambda \in[0,1) \\
& \lim _{\nu \rightarrow \infty} g_{\nu}(\lambda)=\frac{2}{(1-\lambda)^{2}} \quad \text { for } \lambda \in[0,1) .
\end{aligned}
$$

With $\lambda_{0}=0$, real numbers $\lambda_{1}, \lambda_{2}, \ldots, \lambda_{r-1}$ in $(0,1)$ exist such that

$$
\lambda_{\mu+1}\left(1+\lambda_{\mu+1}\right) g_{k}\left(\lambda_{\mu+1}\right)=\left(1+\lambda_{\mu}\right) g_{k}\left(\lambda_{\mu}\right)
$$


for $\mu=0,1, \ldots, r-2$. These numbers are uniquely defined. In fact, the continuous function

$$
h(\lambda)=\lambda(1+\lambda) g_{k}(\lambda)
$$

vanishes for $\lambda=0$ and has the value $k(k+1)$ for $\lambda=1$, i.e., its value for $\lambda=1$ is larger than the right hand side of (2.17), where we have used the assumption $\lambda_{\mu}<1$ and, for $\nu \in \mathbb{N}$, the monotonicity of $g_{k}$. This gives the existence of a number $\lambda_{\mu+1}$ satisfying (2.17). The uniqueness follows from the fact that the function $h$ is strictly monotone. The numerical procedure to compute the numbers $\lambda_{\mu}$ is easy.

We now show that

$$
\begin{aligned}
& \tau_{\mu}=\left(\lambda_{\mu} \lambda_{\mu+1} \cdots \lambda_{r-1}\right)^{2} \quad \text { for } \mu=1,2, \ldots, r-1, \\
& \sigma_{\mu, \nu}=\left(\frac{g_{\nu}\left(\lambda_{\mu-1}\right)}{g_{k}\left(\lambda_{\mu-1}\right)}\right)^{2} \quad \text { for } \mu=1,2, \ldots, r, \nu=1,2, \ldots, k-1,
\end{aligned}
$$

is a leveled partition of type 1 . Let a rectangle $R=\left[\sigma_{\mu, \nu-1}, \sigma_{\mu, \nu}\right] \times\left[\tau_{\mu-1}, \tau_{\mu}\right]$ be given. Since

$$
\left(g_{\nu}\left(\lambda_{\mu-1}\right)-\lambda_{\mu-1} g_{\nu-1}\left(\lambda_{\mu-1}\right)\right)^{2}=g_{\nu}\left(\lambda_{\mu-1}\right)+g_{\nu-1}\left(\lambda_{\mu-1}\right),
$$

it follows from (2.16) that

$$
d(R)=\frac{\lambda_{\mu} \lambda_{\mu+1} \cdots \lambda_{r-1}}{4\left(1+\lambda_{\mu-1}\right) g_{k}\left(\lambda_{\mu-1}\right)}
$$

which, by using (2.17), gives

$$
d(R)=\frac{\lambda_{1} \lambda_{2} \cdots \lambda_{r-1}}{4 g_{k}(0)}
$$

The optimal segmentation for $r=k=3$ is shown in Figure 4; here, $m_{1}=0.035678$.

\section{Algorithm}

We now describe an algorithm for computing a leveled (hence optimal) type 1 partition of a given rectangle $\Omega=[a, b] \times[c, d]$ w.r.t. any functional $d$ satisfying (2.4)-(2.6) and the corresponding optimal value $m_{1}$, which we assume to be positive. It uses some methods developed in the univariate case by Nürnberger, Sommer, Strauß [13] and Meinardus, Nürnberger, Sommer, Strauß [9].

The method is as follows. We compute iteratively a sequence of partitions $\left\{\Omega_{\mu, \nu, p}\right\}_{p \in \mathbb{N}}$, and simultaneously two sequences of real numbers $\left\{d_{p}^{\min }\right\}_{p \in \mathbb{N}}$ and $\left\{d_{p}^{\max }\right\}_{p \in \mathbb{N}}$, which are monotonously increasing respectively decreasing and converge to $m_{1}$, such that for all $p$, the inclusion

$$
d_{p}^{\min } \leq m_{1} \leq d_{p}^{\max }
$$

holds.

In practice, the algorithm terminates if $d_{p}^{\max }-d_{p}^{\min }<\varepsilon$ for some prescribed tolerance $\varepsilon$. 


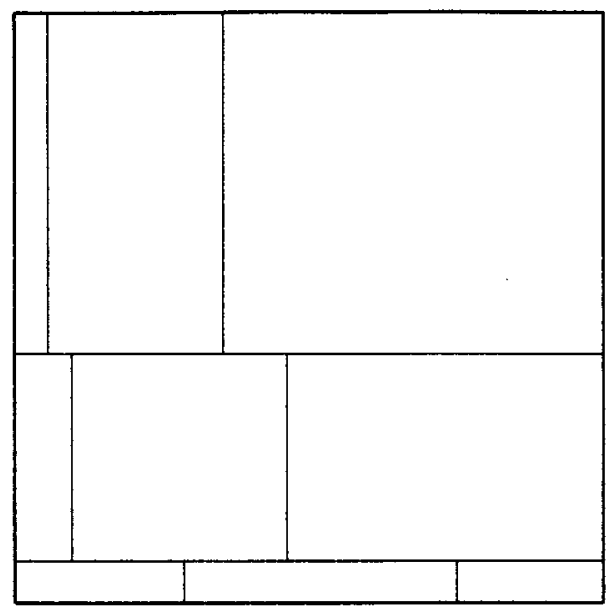

Figure 4. Optimal partition in Example 2.4

\section{Description of the Algorithm}

First Step: In order to get two positive numbers $d_{1}^{\min }$ and $d_{1}^{\max }$ with the property (2.19), compute the values

$$
d\left(\Omega_{\mu, \nu, 1}\right), \quad \nu=1, \ldots, k, \mu=1, \ldots, r
$$

of an arbitrary type 1 partition $\left\{\Omega_{\mu, \nu, 1}\right\}$ of $\Omega$. According to Theorem 2.2, the numbers

$$
\begin{aligned}
d_{1}^{\min } & :=\min _{\mu, \nu} d\left(\Omega_{\mu, \nu, 1}\right) \quad \text { and } \\
d_{1}^{\max } & :=\max _{\mu, \nu} d\left(\Omega_{\mu, \nu, 1}\right)
\end{aligned}
$$

satisfy (2.19). We may assume that $d_{1}^{\min }>0$.

\section{$p^{\text {th }}$ Step:}

a. Set $d_{p}:=\sqrt{d_{p}^{\min } d_{p}^{\max }}$. If $d_{p}^{\max }-d_{p}^{\min }<\varepsilon$, then stop.

b. For $\mu=1,2, \ldots$, determine a strip $J_{\mu, p}=[a, b] \times\left[\tau_{\mu-1, p}, \tau_{\mu, p}\right]$, such that

$$
d\left(\Omega_{\mu, 1, p}\right)=\cdots=d\left(\Omega_{\mu, k, p}\right)=d_{p}
$$

Let $\widetilde{\mu} \leq r-1$ be the maximal index such that this is possible, and put $\tau_{\widetilde{\mu}+1, p}:=\cdots=$ $\tau_{r, p}$.

c. Compute a leveled partition $\left\{\Omega_{r, 1, p}, \ldots, \Omega_{r, k, p}\right\}$ of the strip $J_{r, p}$, and set

$$
c_{p}:=d\left(\Omega_{r, 1, p}\right)=\cdots=d\left(\Omega_{r, k, p}\right) .
$$


d. If $c_{p}=d_{p}$, then stop.

If $c_{p}<d_{p}$, set

$$
\begin{aligned}
d_{p+1}^{\min } & :=\max \left\{d_{p}^{\min }, c_{p}\right\} \\
d_{p+1}^{\max } & :=\min \left\{d_{p}^{\max }, d_{p}\right\}
\end{aligned}
$$

if $c_{p}>d_{p}$, set

$$
\begin{aligned}
d_{p+1}^{\min } & :=\max \left\{d_{p}^{\min }, d_{p}\right\} \\
d_{p+1}^{\max } & :=\min \left\{d_{p}^{\max }, c_{p}\right\}
\end{aligned}
$$

and proceed with the $(p+1)^{\text {st }}$ step.

\section{Details of the Algorithm}

Step b: For simplicity, we omit the index $p$ and set $\mu=1$. The computation of the strip $J_{1}:=[a, b] \times\left[\tau_{0}, \tau_{1}\right]$, i.e., of the parameter $\tau_{1}$, such that

$$
d\left(\Omega_{1,1}\right)=\cdots=d\left(\Omega_{1, k}\right)=d,
$$

is as follows.

Compute a sequence $\left\{\tau_{1, n}\right\}$ such that $\tau_{1, n} \stackrel{n \rightarrow \infty}{\longrightarrow} \tau_{1}$. To do this, determine $\tau_{1,1}$ and $\Omega_{1,1}^{1}, \Omega_{1,2}^{1}, \ldots$, such that

$$
d\left(\Omega_{1,1}^{1}\right)=d\left(\Omega_{1,2}^{1}\right)=\cdots=d
$$

and

$$
d\left(\Omega_{1, k}^{1}\right) \leq d
$$

Here, $\Omega_{1,1}^{1} \cup \cdots \cup \Omega_{1, k}^{1}=[a, b] \times\left[\tau_{0}, \tau_{1,1}\right]$.

Moreover, determine $\tau_{1,2}$ and $\Omega_{1,1}^{2}, \Omega_{1,2}^{2}, \ldots$, such that

$$
d\left(\Omega_{1,1}^{2}\right)=d\left(\Omega_{1,2}^{2}\right)=\cdots=d
$$

and

$$
d\left(\Omega_{1, k}^{2}\right) \geq d
$$

Here, $\Omega_{1,1}^{2} \cup \cdots \cup \Omega_{1, k}^{2}=[a, b] \times\left[\tau_{0}, \tau_{1,2}\right]$.

This implies

$$
\tau_{1,1} \leq \tau_{1} \leq \tau_{1,2}
$$


Set

$$
\tau_{1,3}=\frac{1}{2}\left(\tau_{1,1}+\tau_{1,2}\right)
$$

and determine $\Omega_{1,1}^{3}, \Omega_{1,2}^{3}, \ldots$, such that

$$
d\left(\Omega_{1,1}^{3}\right)=d\left(\Omega_{1,2}^{3}\right)=\cdots=d .
$$

If $d\left(\Omega_{1, k}^{3}\right) \leq d$, then $\tau_{1,3} \leq \tau_{1} \leq \tau_{1,2}$ and set $\tau_{1,4}=\frac{1}{2}\left(\tau_{1,2}+\tau 1,3\right)$, if $d\left(\Omega_{1, k}^{3}\right) \geq d$, then $\tau_{1,1} \leq \tau_{1} \leq \tau_{1,3}$ and set $\tau_{1,4}=\frac{1}{2}\left(\tau_{1,1}+\tau_{1,3}\right)$. Proceed by induction.

Step c: For simplicity, we omit the indices $p$ and $r$. The computation of the leveled partition $\left\{\Omega_{1}, \ldots, \Omega_{k}\right\}$ of a strip $J$ with optimal vallue $c$ follows the same principle as used in the algorithm itself: Given two bounds $c_{n}^{\min } \leq c \leq c_{n}^{\max }$, set, in the $n^{\text {th }}$ step,

$$
\delta_{n}=\sqrt{c_{n}^{\min } c_{n}^{\max }}
$$

Determine rectangles $\Omega_{1}^{n}, \Omega_{2}^{n}, \ldots$, such that

$$
d\left(\Omega_{1}^{n}\right)=d\left(\Omega_{2}^{n}\right)=\cdots=\delta_{n},
$$

and compute $\gamma_{n}:=d\left(\Omega_{k}^{n}\right)$.

If $\gamma_{n}=\delta_{n}$, then stop.

If $\gamma_{n}<\delta_{n}$, set

$$
\begin{aligned}
c_{n+1}^{\min } & :=\max \left\{c_{n}^{\min }, \gamma_{n}\right\} \\
c_{n+1}^{\max } & :=\min \left\{c_{n}^{\max }, \delta_{n}\right\},
\end{aligned}
$$

if $\gamma_{n}>\delta_{n}$, set

$$
\begin{aligned}
& c_{n+1}^{\min }:=\max \left\{c_{n}^{\min }, \delta_{n}\right\} \\
& c_{n+1}^{\max }:=\min \left\{c_{n}^{\max }, \gamma_{n}\right\},
\end{aligned}
$$

and proceed by induction.

The convergence of the algorithm is proved in the following

\section{Theorem 2.5.}

(i) For each $p \in \mathbb{N}$, the relation

$$
0<\left(\frac{d_{1}^{\min }}{d_{1}^{\max }}\right)^{\frac{1}{2^{p}}} \leq \cdots \leq\left(\frac{d_{p}^{\min }}{d_{p}^{\max }}\right)^{\frac{1}{2}} \leq\left(\frac{d_{p+1}^{\min }}{d_{p+1}^{\max }}\right) \leq 1
$$

holds. 
(ii) For each $p \in \mathbb{N}$, the inclusion

$$
d_{p}^{\min } \leq m_{1} \leq d_{p}^{\max }
$$

holds.

(iii) We have

$$
\lim _{p \rightarrow \infty} d_{p}^{\min }=\lim _{p \rightarrow \infty} d_{p}^{\max }=m_{1}
$$

Remark 2.6. In connection with problems of best approximation, the numbers $d_{p}^{\min }$ and $d_{p}^{\max }$ are typically of the form $d_{p}^{\min }=10^{\alpha_{p}}$ and $d_{p}^{\max }=10^{\beta_{p}}$ with $\alpha_{p} \leq \beta_{p} \ll 0$. Then, Theorem 2.5 implies a fast convergence of the algorithm, since it follows from (i) that

$$
\left|\alpha_{p+1}-\beta_{p+1}\right| \leq \frac{1}{2}\left|\alpha_{p}-\beta_{p}\right|
$$

for all $p$.

Proof of Theorem 2.5. From the definition of $d_{p+1}^{\min }$ and $d_{p+1}^{\max }$ in Step 4, it follows directly that

$$
\frac{d_{p+1}^{\min }}{d_{p+1}^{\max }} \geq \frac{d_{p}^{\min }}{d_{p}}=\frac{d_{p}^{\min }}{\sqrt{d_{p}^{\min } d_{p}^{\max }}}
$$

if $c_{p} \leq d_{p}$, and

$$
\frac{d_{p+1}^{\min }}{d_{p+1}^{\max }} \geq \frac{d_{p}}{d_{p}^{\max }}=\frac{\sqrt{d_{p}^{\min } d_{p}^{\max }}}{d_{p}^{\max }}
$$

if $c_{p} \geq d_{p}$. Since $0<d_{p}^{\min } \leq d_{p}^{\max }$ for all $p$, relation (i) is proved. Moreover, (ii) is obvious.

Since $0<d_{p}^{\min } \leq d_{p+1}^{\min } \leq m_{1} \leq d_{p+1}^{\max } \leq d_{p}^{\max }$ for all $p$, we see that

$$
\lim _{p \rightarrow \infty} d_{p}^{\min }=: A \quad \text { and } \quad \lim _{p \rightarrow \infty} d_{p}^{\max }=: B
$$

exist and satisfy

$$
A \leq m_{1} \leq B
$$

It follows from (i) that $\frac{d_{p}^{\min }}{d_{p}^{\max }} \stackrel{p \rightarrow \infty}{\longrightarrow} 1$, hence $A=B$, and so (2.21) implies statement (iii).

\section{Numerical Results}

We give some results on optimal partitions of type 1 on the square $[0,1]^{2}$. We consider the functional

$$
d(R)=\frac{3}{8 \sqrt{\left(x+y+\frac{1}{100}\right)^{5}}} \cdot \max \left\{\left(\xi_{2}-\xi_{1}\right)^{3},\left(\eta_{2}-\eta_{1}\right)^{3}\right\}
$$


where $R=\left[\xi_{1}, \xi_{2}\right] \times\left[\eta_{1}, \eta_{2}\right]$. Note that this functional reflects the error in best approximation of the function

$$
f(x, y)=\sqrt{x+y+\frac{1}{100}}
$$

(cf. (3.2)). For $r=k=6$, the leveled type 1 partition computed by the algorithm is shown in Figure 5.

Our numerical experience shows that the algorithm works efficiently if we use the functional $\tilde{d}(R)=\|f-P(f)\|_{R}$, where $P(f)$ interpolates $f$ at $(m+1)^{2}$ uniformly distributed points in $R$, although $\widetilde{d}$ does not satisfy (2.4) in general. To illustrate this, we show in Figure 6 the leveled type 1 partition w.r.t. $\tilde{d}$ for $m=2$. By comparing Figures 5 and 6 we see that the optimal partitions for both functionals are almost the same.

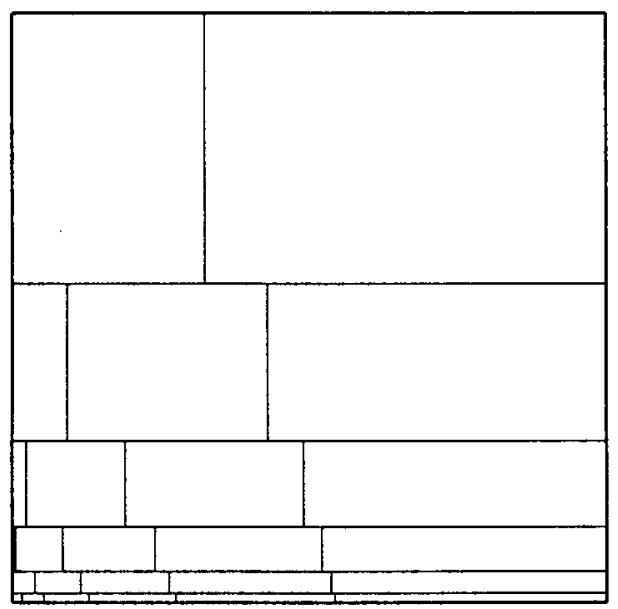

Figure 5.

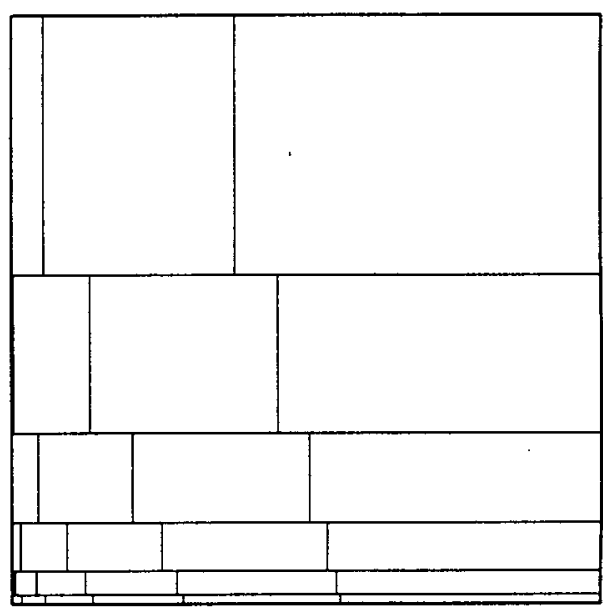

Figure 6.

\section{Partitions of Type 3}

Optimality criteria for type 3 partitions differ very much from that for type 1 and type 2 . In general, for type 3, no leveled partition exists and, on the other hand, if for a partition $\left\{\Omega_{\mu, \nu}\right\}$ all values $d\left(\Omega_{\mu, \nu}\right)$ except one are the same, this still does not imply optimality. We illustrate this behaviour by two examples in the case $r=k=2$.

Example 3.1. Let $\Omega=[0,1] \times[0,1]$ and a function $F: \Omega \rightarrow \mathbb{R}$ be given. We consider the functional $d$ defined by

$$
d(R)=\|F\|_{R} \cdot \max \left\{\left(\xi_{2}-\xi_{1}\right)^{m+1},\left(\eta_{2}-\eta_{1}\right)^{m+1}\right\}
$$

for all subrectangles $R=\left[\xi_{1}, \xi_{2}\right] \times\left[\eta_{1}, \eta_{2}\right]$ of $\Omega$. (Compare the functional $d_{2}$ in $(3.2)$.) 
We first consider the case when $F(x, y)=|x+y-1|$. For the equidistant partition, we have $d\left(\Omega_{1,1}\right)=d\left(\Omega_{2,2}\right)=\left(\frac{1}{2}\right)^{m+1}$ and $d\left(\Omega_{1,2}\right)=d\left(\Omega_{2,1}\right)=\left(\frac{1}{2}\right)^{m+2}$. It is easy to verify that this partition is the unique optimal partition of type 3 but it is not leveled.

We now consider the case when $F \equiv 1$. For $\sigma_{1}=\tau_{1}<\frac{1}{2}$, all values $d\left(\Omega_{\mu, \nu}\right)=\left(1-\sigma_{1}\right)^{m+1}$ are the same except that $d\left(\Omega_{1,1}\right)=\sigma_{1}^{m+1}$. But these partitions are not optimal, since the equidistant partition is the unique optimal partition of type 3 with $m_{3}=\left(\frac{1}{2}\right)^{m+1}$.

Despite these difficulties, we describe an approach to determine optimal type 3 partitions for functionals $d$ which arise from piecewise polynomial approximation. This is done by analyzing the relations between optimal partitions of type 1 respectively type 2, and type 3 . We denote by $\Pi_{m}=\operatorname{span}\left\{1, t, \ldots, t^{m}\right\}$ the space of univariate polynomials of degree $m$ and by $\Pi_{m} \otimes \Pi_{m}=\operatorname{span}\left\{x^{\mu} y^{\nu}: \mu, \nu \in \mathbb{N}_{0}, \mu, \nu \leq m\right\}$ the space of tensor product polynomials of degree $m$.

Let a sufficiently differentiable function $f \in C(\Omega)$ and a subrectangle $R=\left[\xi_{1}, \xi_{2}\right] \times\left[\eta_{1}, \eta_{2}\right]$ of $\Omega$ be given. Since no general methods are available in the literature for computing $\rho\left(f, \Pi_{m} \otimes \Pi_{m}\right)_{R}$, we replace this value by the value $d(R)$ of some appropriately chosen functional $d$.

We define two functionals of this type. The first one replaces the value $\rho\left(f, \Pi_{m} \otimes \Pi_{m}\right)_{R}$ by the maximum of the univariate minimal deviations

$$
\rho\left(f, \Pi_{m}\right)_{I}=\min _{p \in \Pi_{m}}\|f-p\|_{I}
$$

for all horizontal and vertical line segments $I$ in $R$. We define

$$
\begin{aligned}
d_{1}(R) & :=d_{1}(f, R) \\
& :=\max \left\{\max _{x \in\left[\xi_{1}, \xi_{2}\right]} \rho\left(f, \Pi_{m}\right)_{I_{x}(R)}, \max _{y \in\left[\eta_{1}, \eta_{2}\right]} \rho\left(f, \Pi_{m}\right)_{I_{y}(R)}\right\}
\end{aligned}
$$

where $I_{x}:=I_{x}(R)=\{(\xi, \eta) \in R: \xi=x\}$, and $I_{y}:=I_{y}(R)=\{(\xi, \eta) \in R: \eta=y\}$. Obviously, the value $d_{1}(R)$ is a lower bound for $\rho\left(f, \Pi_{m} \otimes \Pi_{m}\right)$.

The second functional is up to some constant an upper bound for the error $\|f-P(f)\|_{R}$ and therefore, for $\rho\left(f, \Pi_{m} \otimes \Pi_{m}\right)_{R}$. Here, $P(f)$ is the unique polynomial in $\Pi_{m} \otimes \Pi_{m}$ which interpolates $f$ at $(m+1)^{2}$ uniformly distributed points in $R$. We define the functional $d_{2}$ by

$$
\begin{aligned}
d_{2}(R) & :=d_{2}(f, R) \\
& :=\max \left\{\left\|f_{x^{m+1}}\right\|_{R} \cdot\left(\xi_{2}-\xi_{1}\right)^{m+1},\left\|f_{y^{m+1}}\right\|_{R} \cdot\left(\eta_{2}-\eta_{1}\right)^{m+1}\right\},
\end{aligned}
$$

where $f_{x}$ and $f_{y}$ denote the partial derivatives of $f$.

For these functionals, the estimate

$$
d_{1}(f, R) \leq \rho\left(f, \Pi_{m} \otimes \Pi_{m}\right)_{R} \leq\|f-P(f)\|_{R} \leq C d_{2}(f, R)
$$


holds, where $C$ is some positive constant.

The last inequality follows from the next result in which we give an upper bound for the interpolation error $\|f-P(f)\|_{R}$. This estimate differs from the known upper bounds in which an additional term $\left\|f_{x^{m+1} y^{m+1}}\right\|_{R}\left(\xi_{2}-\xi_{1}\right)^{m+1}\left(\eta_{2}-\eta_{1}\right)^{m+1}$ appears (see e.g. $[2$, p. 87]).

Theorem 3.2. For each function $f \in C^{m+1}(\Omega)$, a constant $K>0$ exists such that

$$
\|f-P(f)\|_{R} \leq K\left(\left\|f_{x^{m+1}}\right\|_{R}\left(\xi_{2}-\xi_{1}\right)^{m+1}+\left\|f_{y^{m+1}}\right\|_{R}\left(\eta_{2}-\eta_{1}\right)^{m+1}\right),
$$

where $K$ is independent of $\xi_{1}, \xi_{2}, \eta_{1}, \eta_{2}$ and $f$.

Proof. We denote by $\left\{\left(x_{i}, y_{j}\right): i, j=0, \ldots, m\right\}$ the set of uniformly distributed interpolation points in $R$. Moreover, we set $h_{1}:=\xi_{2}-\xi_{1}, h_{2}:=\eta_{2}-\eta_{1}$ and consider for $\bar{x} \in\left[\xi_{1}, \xi_{2}\right]$, the line segment

$$
I_{\bar{x}}:=\{(\xi, \eta) \in R: \xi=\bar{x}\}
$$

Let $p \in \Pi_{m}$ denote the unique univariate polynomial which interpolates $f$ at the points $\left\{\left(\bar{x}, y_{j}\right)\right\}_{j=0, \ldots, m}$. It follows that

$$
\|f-P(f)\|_{I_{\bar{x}}} \leq\|f-p\|_{I_{\bar{x}}}+\|p-P(f)\|_{I_{\bar{x}}}
$$

and the univariate interpolation theory implies that

$$
\|f-p\|_{I_{\bar{x}}} \leq \frac{1}{(m+1) !}\left\|f_{y^{m+1}}\right\|_{I_{\bar{x}}} \cdot h_{2}^{m+1} .
$$

Now, we consider the univariate polynomial

$$
q(y):=p(y)-P(f)(\bar{x}, y)
$$

Since $p$ interpolates $f$ at the points $\left\{\left(\bar{x}, y_{j}\right)\right\}$, it follows again from the univariate theory that

$$
\begin{aligned}
\left|q\left(y_{j}\right)\right| & =\left|f\left(\bar{x}, y_{j}\right)-P(f)\left(\bar{x}, y_{j}\right)\right| \\
& \leq \frac{1}{(m+1) !}\left\|f_{x^{m+1}}\right\|_{I_{y_{j}}} \cdot h_{1}^{m+1} \\
& \leq \frac{1}{(m+1) !}\left\|f_{x^{m+1}}\right\|_{R} \cdot h_{1}^{m+1}
\end{aligned}
$$

for $j=0, \ldots, m$, where

$$
I_{y_{j}}:=\left\{(\xi, \eta): \eta=y_{j}\right\}
$$

We now write the polynomial $q$ in Newton form, i.e.,

$$
q(y)=\sum_{\mu=0}^{m} \Delta\left(y_{0}, \ldots, y_{\mu} ; q\right)\left(y-y_{0}\right) \ldots\left(y-y_{\mu-1}\right)
$$


where $\Delta$ denotes the usual divided difference.

Using the recurrence relation for divided differences and inequality (3.8), it is easily shown by induction that for each $\mu \in\{0, \ldots, m\}$ a constant $K(\mu)>0$ exists such that

$$
\left|\Delta\left(y_{0}, \ldots, y_{\mu} ; q\right)\right| \leq K(\mu) \cdot\left\|f_{x^{m+1}}\right\|_{R} \cdot h_{1}^{m+1} \cdot h_{2}^{-\mu} .
$$

This implies, using (3.9), that

$$
\|q\|_{I_{\bar{x}}} \leq \widetilde{K} \cdot\left\|f_{x^{m+1}}\right\|_{R} \cdot h_{1}^{m+1}
$$

with some constant $\widetilde{K}>0$.

Since $R=\bigcup_{\bar{x} \in\left[\xi_{1}, \xi_{2}\right]} I_{\bar{x}}$, the combination of (3.5), (3.6) and (3.10) completes the proof of Theorem 3.2 .

Remark 3.3. It is also possible to prove an analogous result for the case of Hermite interpolation by tensor product polynomials.

From now on, $d$ denotes the functional $d_{1}$ or $d_{2}$, defined in (3.1) and (3.2). We now investigate type 3 partitions for these functionals. First we describe a method for constructing an optimal type 3 partition by using an optimal type 1 partition.

\section{Algorithm}

Step 1: We construct a leveled partition of type 1 and fix the corresponding $\tau$-parameters $\bar{\tau}_{0} \leq \cdots \leq \bar{\tau}_{r}$ (by applying the algorithm in Section 2).

Step 2: We consider the univariate functional

$$
\bar{d}\left(\xi_{1}, \xi_{2}\right):=\max _{\mu=1, \ldots, r} d\left(\left[\xi_{1}, \xi_{2}\right] \times\left[\bar{\tau}_{\mu-1}, \bar{\tau}_{\mu}\right]\right)
$$

where $a \leq \xi_{1} \leq \xi_{2} \leq b$. We construct a leveled partition for this functional $\bar{d}$, i.e., we compute parameters $\bar{\sigma}_{0} \leq \cdots \leq \bar{\sigma}_{k}$ such that

$$
\bar{d}\left(\bar{\sigma}_{\nu-1}, \bar{\sigma}_{\nu}\right)=\bar{d}\left(\bar{\sigma}_{\nu}, \bar{\sigma}_{\nu+1}\right), \quad \nu=1, \ldots, k-1
$$

(by applying the algorithm in Section 2). We denote the optimal value in (3.12) by $\bar{m}$.

The next result shows that in this way, we obtain an optimal partition of type 3 .

Theorem 3.4. The partition $\left\{\bar{\Omega}_{\mu, \nu}\right\}$, defined by

$$
\bar{\Omega}_{\mu, \nu}:=\left[\bar{\sigma}_{\nu-1}, \bar{\sigma}_{\nu}\right] \times\left[\bar{\tau}_{\mu-1}, \bar{\tau}_{\mu}\right], \quad \mu=1, \ldots, r, \nu=1, \ldots, k,
$$

is an optimal partition of type 3. 
We also show that the optimal value of type 3 partitions is equal to one of the optimal values of type 1 and type 2 partitions.

Theorem 3.5. We have

$$
m_{3}=\max \left\{m_{1}, m_{2}\right\}
$$

where $m_{i}$ is the optimal value for partitions of type $i, i=1,2,3$.

Finally, we establish a connection between the three types of optimal partitions. It is shown that optimal partitions of type 1 and type 2 yield an optimal type 3 partition.

Theorem 3.6. The horizontal lines of an optimal type 1 partition combined with the vertical lines of an optimal type 2 partition yield an optimal type 3 partition.

Proof of Theorems 3.4-3.6. Let $d=d_{1}$ or $d=d_{2}$.

It follows from the definition of $\bar{m}$ that

$$
d\left(\bar{\Omega}_{\mu, \nu}\right) \leq \bar{m}
$$

for all $\mu$ and $\nu$. Since $\left\{\bar{\Omega}_{\mu, \nu}\right\}$ is a partition of type 3 , the inequalities

$$
m_{1} \leq m_{3} \leq \bar{m} \quad \text { and } \quad m_{2} \leq m_{3} \leq \bar{m}
$$

follow.

We now distinguish two cases:

Case 1. $m_{1}=\bar{m}$

In this case $m_{1}=m_{3}$ as a consequence of (3.13), and Theorems 3.4 and 3.5 are proved.

Case 2. $m_{1}<\bar{m}$

We first consider the functional $d=d_{2}$. Since $\left\{\bar{\sigma}_{0}, \ldots, \bar{\sigma}_{k}\right\}$ is a leveled parameter set w.r.t. the functional $\bar{d}$ (cf. (3.12)), there exist indices $\mu_{1}, \ldots, \mu_{k}$, such that

$$
\bar{m}=\bar{d}\left(\bar{\sigma}_{\nu-1}, \bar{\sigma}_{\nu}\right)=d\left(\dot{\bar{\Omega}}_{\mu_{\nu}, \nu}\right) \quad \text { for } \nu=1, \ldots, k .
$$

Moreover, since the parameters $\bar{\tau}_{0}, \ldots, \bar{\tau}_{r}$ belong to a leveled type 1 partition, we see that for each $\mu=1, \ldots, r$ and $z \in[a, b] \times\left[\bar{\tau}_{\mu-1}, \bar{\tau}_{\mu}\right]$,

$$
\left|f_{y^{m+1}}(z)\right| \cdot\left(\bar{\tau}_{\mu}-\bar{\tau}_{\mu-1}\right)^{m+1} \leq m_{1} \text {. }
$$

Therefore

$$
\begin{aligned}
\bar{m} & =d\left(\bar{\Omega}_{\mu_{\nu}, \nu}\right) \\
& >m_{1} \\
& \geq\left|f_{y^{m+1}}(z)\right| \cdot\left(\bar{\tau}_{\mu_{\nu}}-\bar{\tau}_{\mu_{\nu}-1}\right)^{m+1}
\end{aligned}
$$

is valid for all $z \in[a, b] \times\left[\bar{\tau}_{\mu_{\nu}-1}, \bar{\tau}_{\mu_{\nu}}\right]$ and $\nu=1, \ldots, k$. 
This implies that

$$
\begin{aligned}
&\left\|f_{y^{m+1}}\right\|_{\bar{\Omega}_{\mu_{\nu}, \nu}} \cdot\left(\bar{\tau}_{\mu_{\nu}}-\bar{\tau}_{\mu_{\nu}-1}\right)^{m+1} \\
& \quad<d\left(\bar{\Omega}_{\mu_{\nu}, \nu}\right) \\
& \quad=\max \left\{\left\|f_{x^{m+1}}\right\|_{\bar{\Omega}_{\mu_{\nu}, \nu}} \cdot\left(\bar{\sigma}_{\nu}-\bar{\sigma}_{\nu-1}\right)^{m+1},\left\|f_{y^{m+1}}\right\|_{\bar{\Omega}_{\mu_{\nu}, \nu}} \cdot\left(\bar{\tau}_{\mu_{\nu}}-\bar{\tau}_{\mu_{\nu}-1}\right)^{m+1}\right\}
\end{aligned}
$$

and so

$$
d\left(\bar{\Omega}_{\mu_{\nu}, \nu}\right)=\left\|f_{x^{m+1}}\right\|_{\bar{\Omega}_{\mu_{\nu}, \nu}} \cdot\left(\bar{\sigma}_{\nu}-\bar{\sigma}_{\nu-1}\right)^{m+1}
$$

for $\nu=1, \ldots, k$.

For $\nu=1, \ldots, k$, let $z_{\mu_{\nu}, \nu} \in \bar{\Omega}_{\mu_{\nu}, \nu}$ be a point where the norm in (3.16) is attained, i.e.,

$$
\left|f_{x^{m+1}}\left(z_{\mu_{\nu}, \nu}\right)\right|=\left\|f_{x^{m+1}}\right\|_{\bar{\Omega}_{\mu_{\nu}, \nu}} .
$$

Let $\left\{\Omega_{\mu, \nu}\right\}$ be an arbitrary type 2 partition with parameters $\left\{\sigma_{0}, \ldots, \sigma_{k}\right\}$. There exists an index $n \in\{1, \ldots, k\}$ such that

$$
\left[\bar{\sigma}_{n-1}, \bar{\sigma}_{n}\right] \subset\left[\sigma_{n-1}, \sigma_{n}\right]
$$

(cf. the proof of Theorem 2.2).

Moreover, there is some $\Omega_{\mu, n}$ such that

$$
z_{\mu_{n}, n} \in \Omega_{\mu, n}
$$

Now it follows from (3.14), (3.16), (3.18) and (3.19) that

$$
\begin{aligned}
d\left(\Omega_{\mu, n}\right) & \geq\left\|f_{x^{m+1}}\right\|_{\Omega_{\mu, n}} \cdot\left(\sigma_{n}-\sigma_{n-1}\right)^{m+1} \\
& \geq\left|f_{x^{m+1}}\left(z_{\mu_{n}, n}\right)\right| \cdot\left(\sigma_{n}-\sigma_{n-1}\right)^{m+1} \\
& \geq\left\|f_{x^{m+1}}\right\|_{\bar{\Omega}_{\mu_{n}, n}} \cdot\left(\bar{\sigma}_{n}-\bar{\sigma}_{n-1}\right)^{m+1} \\
& =d\left(\bar{\Omega}_{\mu_{n}, n}\right) \\
& =\bar{m} .
\end{aligned}
$$

Since $\left\{\Omega_{\mu, \nu}\right\}$ is an arbitrary type 2 partition, and since $\left\{\bar{\Omega}_{\mu, \nu}\right\}$ is also of type 2 , it follows that $\bar{m}=m_{2}$, hence $m_{3}=\bar{m}$. This proves Theorems 3.4 and 3.5 for $d=d_{2}$.

Now let $d=d_{1}$. Since the parameters $\bar{\tau}_{0}, \ldots, \bar{\tau}_{r}$ belong to a leveled type 1 partition, we have for each $\mu=1, \ldots, r$ and $x \in[a, b]$,

$$
\rho\left(f, \Pi_{m}\right)_{I_{x}\left([a, b] \times\left[\bar{\tau}_{\mu-1}, \bar{\tau}_{\mu}\right]\right)} \leq m_{1},
$$

hence

$$
\begin{aligned}
\bar{m} & =d\left(\bar{\Omega}_{\mu_{\nu}, \nu}\right) \\
& >m_{1} \\
& \geq \rho\left(f, \Pi_{m}\right)_{I_{x}\left([a, b] \times\left[\bar{\tau}_{\mu_{\nu}-1}, \bar{\tau}_{\mu_{\nu}}\right]\right)}
\end{aligned}
$$


for $\nu=1, \ldots, k$. It follows that

$$
d\left(\bar{\Omega}_{\mu_{\nu}, \nu}\right)=\max _{y \in\left[\bar{\tau}_{\mu_{\nu}-1}, \bar{\tau}_{\mu_{\nu}}\right]} \rho\left(f, \Pi_{m}\right)_{I_{y}\left(\bar{\Omega}_{\mu_{\nu}, \nu}\right)}
$$

for $\nu=1, \ldots, k$.

Let $I_{y_{\mu_{\nu}}} \subset \bar{\Omega}_{\mu_{\nu}, \nu}$ be a line segment where the maximum in (3.21) is attained, i.e.,

$$
d\left(\bar{\Omega}_{\mu_{\nu}, \nu}\right)=\rho\left(f, \Pi_{m}\right)_{I_{y_{\nu}}\left(\bar{\Omega}_{\mu_{\nu}, \nu}\right)} .
$$

Let $\left\{\Omega_{\mu, \nu}\right\}$ be an arbitrary type 2 partition, and $n$ an index such that (3.18) holds. Then there is some $\Omega_{\mu, n}$ such that

$$
I_{y_{\mu_{n}}} \subset \Omega_{\mu, n}
$$

It follows from (3.14), (3.18), (3.22) and (3.23) that

$$
\begin{aligned}
d\left(\Omega_{\mu, n}\right) & \geq \max _{y \in\left[\tau_{\mu-1}, \tau_{\mu}\right]} \rho\left(f, \Pi_{m}\right)_{I_{y}\left(\Omega_{\mu, n}\right)} \\
& \geq \rho\left(f, \Pi_{m}\right)_{I_{y_{n}}\left(\Omega_{\mu, n}\right)} \\
& \geq \rho\left(f, \Pi_{m}\right)_{I_{y_{\mu_{n}}}\left(\bar{\Omega}_{\mu, n}\right)} \\
& =d\left(\bar{\Omega}_{\mu_{n}, n}\right) \\
& =\bar{m}
\end{aligned}
$$

As above, it follows that $\bar{m}=m_{2}=m_{3}$. This completes the proof of Theorems 3.4 and 3.5.

We now prove Theorem 3.6. Let

$$
\left\{(\xi, \eta): \eta=\tau_{\mu}^{*}\right\}_{\mu=0, \ldots, r}
$$

be the horizontal lines of an optimal type 1 partition, and

$$
\left\{(\xi, \eta): \xi=\sigma_{\nu}^{*}\right\}_{\nu=0, \ldots, k}
$$

the vertical lines of an optimal type 2 partition. We have to show that $\left\{\Omega_{\mu, \nu}^{*}\right\}$, where

$$
\Omega_{\mu, \nu}^{*}:=\left[\sigma_{\nu-1}^{*}, \sigma_{\nu}^{*}\right] \times\left[\tau_{\mu-1}^{*}, \tau_{\mu}^{*}\right]
$$

$\nu=1, \ldots, k, \mu=1, \ldots, r$, is an optimal type 3 partition.

To do this, let $\mu \in\{1, \ldots, r\}$ be fixed. Then, for each $z \in J_{\mu}^{*}$,

$$
J_{\mu}^{*}:=[a, b] \times\left[\tau_{\mu-1}^{*}, \tau_{\mu}^{*}\right]
$$

we have

$$
\left|f_{y^{m+1}}(z)\right| \cdot\left(\tau_{\mu}^{*}-\tau_{\mu-1}^{*}\right)^{m+1} \leq m_{1}
$$


if $d=d_{2}$, and for each $x \in[a, b]$

$$
\rho\left(f, \Pi_{m}\right)_{I_{x}\left(J_{\mu}^{*}\right)} \leq m_{1}
$$

if $d=d_{1}$.

Analogously, for each $\nu \in\{1, \ldots, k\}$ and $z \in J_{\nu}^{*}$,

$$
J_{\nu}^{*}:=\left[\sigma_{\nu-1}^{*}, \sigma_{\nu}^{*}\right] \times[c, d],
$$

we have

$$
\left|f_{x^{m+1}}(z)\right| \cdot\left(\sigma_{\nu}^{*}-\sigma_{\nu-1}^{*}\right)^{m+1} \leq m_{2},
$$

if $d=d_{2}$, and for each $y \in[c, d]$,

$$
\rho\left(f, \Pi_{m}\right)_{I_{y}\left(J_{\nu}^{*}\right)} \leq m_{2}
$$

if $d=d_{1}$.

Since $m_{1} \leq m_{3}$ and $m_{2} \leq m_{3}$, equations (3.24) and (3.26) (respectively (3.25) and (3.27)) imply that for all $\mu$ and $\nu$

$$
\left\|f_{x^{m+1}}\right\|_{\Omega_{\mu, \nu}^{*}}\left(\sigma_{\nu}^{*}-\sigma_{\nu-1}^{*}\right)^{m+1} \leq m_{3} \quad \text { and } \quad\left\|f_{y^{m+1}}\right\|_{\Omega_{\mu, \nu}^{*}}\left(\tau_{\mu}^{*}-\tau_{\mu-1}^{*}\right)^{m+1} \leq m_{3}
$$

respectively

$$
\rho\left(f, \Pi_{m}\right)_{I_{y}\left(\Omega_{\mu, \nu}^{*}\right)} \leq m_{3} \quad \text { and } \quad \rho\left(f, \Pi_{m}\right)_{I_{x}\left(\Omega_{\mu, \nu}^{*}\right)} \leq m_{3},
$$

hence $\left\{\Omega_{\mu, \nu}^{*}\right\}$ is an optimal partition of type 3. This completes the proof of Theorems 3.43.6 .

\section{Numerical Results}

Some results concerning optimal type 3 partitions, computed by using the method of Theorem 3.6 , are given.

We continue the example of Section 2 concerning approximation of the square root function

$$
f(x, y)=\sqrt{x+y+\frac{1}{100}} .
$$

The optimal type 3 partition for the functional $d$, defined in (2.22), is shown in Figure 7 .

In a second step, this optimal partition is used for interpolation by tensor product splines from the spaces

$$
S_{2,2}^{i}:=\left\{s \in C^{i}(\Omega):\left.s\right|_{\Omega_{\mu, \nu}} \in \Pi_{2} \otimes \Pi_{2} \text { for all }(\mu, \nu)\right\}
$$




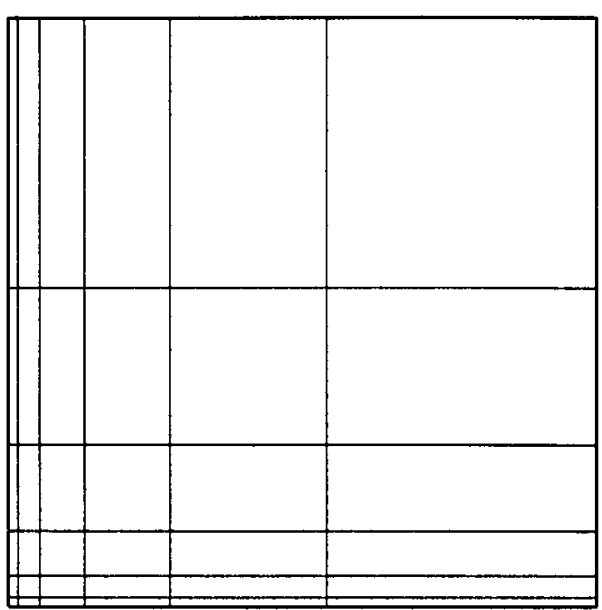

Figure 7.

\begin{tabular}{c|c|c} 
& optimal partition & equidistant partition \\
\hline$S_{2,2}^{0}$ & 0.000635 & 0.01759 \\
\hline$S_{2,2}^{1}$ & 0.000654 & 0.02508
\end{tabular}

Table 1.

for $i=0$ and $i=1$.

The entries of Table 1 are the errors

$$
\|f-s\|_{[0,1]^{2}}
$$

where $s$ is the interpolating spline from $S_{2,2}^{0}$ respectively $S_{2,2}^{1}$, corresponding to the optimal partition respectively to the equidistant partition.

By comparing the errors in both lines of the table, we see that there is a significant reduction of the interpolation error when we use the optimal partition instead of the equidistant one.

Moreover, these errors are compared with the maximum of the errors for piecewise polynomial interpolation at nine equidistant points on each subrectangle of the partition in Figure 5 (respectively Figure 6). The maximal error is 0.000755 (respectively 0.000541) (cf. Table 1), and the errors on each subrectangle are almost the same (respectively the same).

\section{References}

[1] L. Collatz: Einschließungssatz für die Minimalabweichung bei der Segmentapproximation. In: Simp. Intern. Appl. Anal. Fis. Mat., 11-21, 1964. 
[2] F.-J. Delvos, W. Schempp: Boolean Methods in Interpolation and Approximation. Longman, Harlow, 1989.

[3] H. Ehlich, K. Zeller: Cebyšev-Polynome in mehreren Veränderlichen. Math. Zeitschr. 93 (1966), 142-143.

[4] R. Grothmann, H. N. Mhaskar: Detection of singularities using segment approximation. Math. Comp. 59 (1992), 533-540.

[5] C. L. Lawson: Characteristic properties of the segmented rational minimax approximation problem. Numer. Math. 6 (1964), 293-301.

[6] G. Meinardus: Approximation of Functions. Theory and Numerical Methods. Springer, Berlin, Heidelberg, New York, 1967.

[7] G. Meinardus: Optimal partitioning in univariate and multivariate approximation. In: D. Bainov, V. Covachev (eds.), Second International Colloquium on Numerical Analysis, VSP, Utrecht, 123-132, 1993.

[8] G. Meinardus: Some results in segmential approximation. Intern. J. Comp. Math. Appl., to appear.

[9] G. Meinardus, G. Nürnberger, M. Sommer, H. Strauß: Algorithms for piecewise polynomials and splines with free knots. Math. of Comp. 53 (1989), 235-247.

[10] G. Meinardus, G. Walz: Approximation Theory. Springer, Berlin, Heidelberg, New York, in preparation.

[11] G. Nürnberger: Approximation by Spline Functions. Springer, Berlin, Heidelberg, New York, 1989.

[12] G. Nürnberger: Approximation by univariate and bivariate splines. In: D. Bainov, V. Covachev (eds.), Second International Colloquium on Numerical Analysis, VSP, Utrecht, 143-153, 1993.

[13] G. Nürnberger, M. Sommer, H. Strauß: An algorithm for segment approximation. Num. Math. 48 (1986), 463-477.

[14] M. Reimer: On multivariate polynomials of least deviation from zero on the unit cube. J. Approx. Theory 23 (1978), 65-69.

[15] M. Sommer: Segment approximation using linear functionals. In: D. Braess, L. L. Schumaker (eds.), Numerical Methods in Approximation Theory, ISNM 105, Birkhäuser, Basel, 331-346, 1992. 